\title{
ON THE FIRST EIGENVALUE OF NON-ORIENTABLE CLOSED SURFACES
}

\author{
By \\ Katsuhiro YosHIJI
}

\section{Introduction}

Let $(M, g)$ be a 2-dimensional non-orientable closed Riemannian manifold. We study the spectrum of the Laplacian for functions on $(M, g)$. We express it by

$$
\operatorname{Spec}(M, g)=\left\{0=\lambda_{0}<\lambda_{1} \leq \lambda_{2} \leq \lambda_{3} \leq \cdots\right\} .
$$

Let $(\tilde{M}, \tilde{g})$ be the orientable Riemannian double cover of $(M, g)$. Our interest is what properties are preserved between $(M, g)$ and $(\tilde{M}, \tilde{g})$. The positive first eigenvalue $\lambda_{1}(M, g)$ has many geometric informations. We have interests in the influences for the positive first eigenvalue by taking the Riemannian double cover. Generally we have $\lambda_{1}(M, g) \geq \lambda_{1}(\tilde{M}, \tilde{g})$. So we study the difference between $\lambda_{1}(M, g)$ and $\lambda_{1}(\tilde{M}, \tilde{g})$. Especially we find the cases that $\lambda_{1}(M, g)=\lambda_{1}(\tilde{M}, \tilde{g})$ holds good.

It is well-known (cf. [9]) that 2-dimensional closed manifolds are classified as follows.

The Classification Theorem of Closed Surfaces. A closed surface is homeomorphic to one of the following spaces.

$$
\begin{gathered}
S^{2}, T^{2}, \#^{n} T^{2}(n \geq 2): \text { orientable } \\
\boldsymbol{R} \boldsymbol{P}^{2}, \#^{n} \boldsymbol{R} \boldsymbol{P}^{2}(n \geq 2): \text { non-orientable }
\end{gathered}
$$

where $\#^{n} M$ means the connected sum of $n$-copies of a manifold $M$. Moreover the double cover of $\#^{n} \boldsymbol{R} \boldsymbol{P}^{2}(n \geq 2)$ is homeomorphic to $\#^{n-1} T^{2}$.

In this paper we show the following results.

THEOREM A. If $\boldsymbol{M}$ is homeomorphic to $\boldsymbol{R P}^{2}$, then 


$$
\lambda_{1}(M, g)>\lambda_{1}(\tilde{M}, \tilde{g})
$$

for every metric $g$ on $M$.

THEOREM B. If $M$ is homeomorphic to $\#^{n} \boldsymbol{R} \boldsymbol{P}^{2}(n \geq 2)$, there exists a metric $g$ on $M$ such that

$$
\lambda_{1}(M, g)=\lambda_{1}(\tilde{M}, \tilde{g}) .
$$

The author would like to thank J. Takahashi and the referee for useful comments.

\section{Preliminaries}

Let us consider the Riemannian double covering $(\tilde{M}, \tilde{g})$ of $(M, g)$. We define the isometry $J:(\tilde{M}, \tilde{g}) \rightarrow(\tilde{M}, \tilde{g})$ as follows. For each point $p$ of $(M, g)$, let two points $\tilde{p}_{1}, \tilde{p}_{2}$ of $(\tilde{M}, \tilde{g})$ be the fiber of a point $p$ in $(M, g)$. Then we define $J$ by exchanging two points $\tilde{p}_{1}, \tilde{p}_{2}$. Let $E(\lambda), E^{+}(\lambda)$ and $E^{-}(\lambda)$ be the spaces of $C^{\infty}$ functions on $\tilde{M}$ such that

$$
\begin{aligned}
& E(\lambda)=\text { the eigenspace associated with the eigenvalue } \lambda, \\
& \qquad \begin{array}{c}
E^{+}(\lambda)=\{f \in E(\lambda) \mid f \circ J=f\}, \\
E^{-}(\lambda)=\{f \in E(\lambda) \mid f \circ J=-f\} .
\end{array}
\end{aligned}
$$

Proposition (P. Buser [3], p. 306). $E(\lambda)$ is decomposed orthogonally as $E^{+}(\lambda) \oplus E^{-}(\lambda)$.

Since all eigenfunctions on $(M, g)$ are lifted to ones on $(\tilde{M}, \tilde{g})$ canonically, the eigenvalues on $(M, g)$ are in $\operatorname{Spec}(\tilde{M}, \tilde{g})$. The eigenfunctions on $(\tilde{M}, \tilde{g})$ which come from ones on $(M, g)$ are invariant by $J$. Conversely every $f \in E^{+}(\lambda)$ is reduced to the eigenfunction on $(M, g)$. The eigenvalues on $(M, g)$ coincide with the ones on $(\tilde{M}, \tilde{g})$ satisfying $E^{+}(\lambda) \neq\{0\}$.

The eigenfunctions on $(\tilde{M}, \tilde{g})$ which do not come from ones on $(M, g)$ have non-zero components of $E^{-}(\lambda)$ under the above decomposition. Thus we concentrate our attension on the non-zero smallest $\lambda$ such as $E^{-}(\lambda) \neq\{0\}$. We denote it by $v$. Our purpose is to compare $\lambda_{1}(M, g)$ with $v$.

\section{Proof of Theorem A}

In this section the main tool is the nodal domain theorem (cf. [6] and [7]). 
Proposition (S. Y. Cheng [6], p. 186). Let $g$ be any Riemannian metric on $S^{2}$. Then the nodal line of a first eigenfunction is a smooth simple closed curve.

We assume that $(M, g)$ is homeomorphic to $\boldsymbol{R} \boldsymbol{P}^{2}$. Then its Riemannian double cover $(\tilde{M}, \tilde{g})$ is homeomorphic to $S^{2}$. If the first eigenfunction $\varphi$ on $(M, g)$ lifts to the first eigenfunction $\tilde{\varphi}$ on $(\tilde{M}, \tilde{g})$, the nodal set of $\tilde{\varphi}$ is a simple closed curve and the number of the nodal domains is two. We denote one of them by $D$. Since $\pi_{1}(\tilde{M}) \cong\{e\}$, the nodal set $\partial D$ is contractible. Then $D$ is homeomorphic to an open 2-disk. Its boundary $\partial D$ is regularly embedded. Then its closure $\bar{D}$ is homeomorphic to a closed 2-disk. Without loss of generality we take $D$ the positive nodal domain. Since $\tilde{\varphi}$ is invariant by the isometry $J$, we have $J(\bar{D})=\bar{D}$.

We apply the Brouwer's fixed poin theorem (cf. [8] p. 19) to $J: \bar{D} \rightarrow \bar{D}$. Then $J$ has a fixed point. But by the definition of $J$, it does not have any fixed points. It is a contradiction.

\section{Proof of Theorem B}

The first eigenvalue $\lambda_{1}$ is characterized by the Rayleigh quotient, that is,

$$
\lambda_{1}(M, g)=\inf \frac{\int_{M}|\nabla f|^{2} d v}{\int_{M} f^{2} d v}
$$

where $f$ runs over all non-vanishing functions orthogonal to constant functions in $L^{2}(M, g)$.

Our method to prove Proposition B is an analogue of Cheeger's construction in [5] of the deformation of Riemannian metrics $g_{\varepsilon}$ on $S^{2}$ such that $\lambda_{1}\left(S^{2}, g_{\varepsilon}\right)$ converges to 0 as $\varepsilon \rightarrow 0$.

Let us recall the construction.

Step 1. Connect two canonical spheres, $\left(S^{2}, g\right) \#\left(S^{2}, g\right)$, by the tube whose radius is $\varepsilon$ and length is $l$. We express it by $\left(S, g_{\varepsilon}\right)$.

Step 2. We consider the test functions $f_{\varepsilon}$ which is equal to $c$ on the righthand bulb, $-c$ on the left-hand bulb and change linearly from $c$ to $-c$ across tube. We choose $c$ so $\int_{S} f_{\varepsilon}^{2} d v=1$.

Step 3. By the Rayleigh quotient, $\lambda_{1}\left(S, g_{\varepsilon}\right)$ converges to 0 as $\varepsilon \rightarrow 0$.

Now we proceed this method to $\#^{n} \boldsymbol{R} \boldsymbol{P}^{2}$ for $n \geq 2$. We consider $\#^{n} \boldsymbol{R} \boldsymbol{P}^{2}$ as $\left(\#^{n-1} \boldsymbol{R} \boldsymbol{P}^{2}\right) \# \boldsymbol{R} \boldsymbol{P}^{2}$. We take a suitable metric on $\#^{n} \boldsymbol{R} \boldsymbol{P}^{2}$ in such a way that

$$
\frac{\operatorname{Vol}\left(\#^{n-1} \boldsymbol{R} \boldsymbol{P}^{2}\right)}{\operatorname{Vol}\left(\boldsymbol{R} \boldsymbol{P}^{2}\right)}=n-1 .
$$




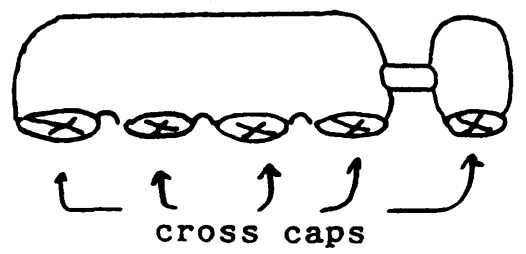

$\left(M, g_{\varepsilon}\right)=\left(\#^{n-1} R P^{2}\right) \# R P^{2}$

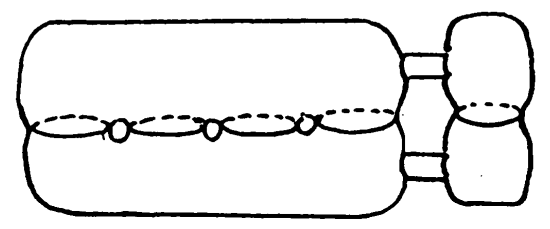

$\left(\tilde{M}, \tilde{g}_{\varepsilon}\right)$

We connect $\#^{n-1} \boldsymbol{R} \boldsymbol{P}^{2}$ and $\boldsymbol{R} \boldsymbol{P}^{2}$ by the tube whose radius is $\varepsilon$ and length is $l$. We denote it by $\left(M, g_{\varepsilon}\right)$. We consider the test functions $f_{\varepsilon}$ which are equal to 1 on $\#^{n-1} \boldsymbol{R} \boldsymbol{P}^{2}$ and $-(n-1)$ on $\boldsymbol{R} \boldsymbol{P}^{2}$, and decrease from 1 to $-(n-1)$ across the tube. We may take $f_{\varepsilon}$ such that they are orthogonal to constant functions in $L^{2}\left(M, g_{\varepsilon}\right)$ and $\left|\nabla f_{\varepsilon}\right| \leq d$ where $d$ is a constant depending only on $l$ and $n$. It follows from the Rayleigh quotient that

$$
\lim _{\varepsilon \rightarrow 0} \lambda_{1}\left(M, g_{\varepsilon}\right)=0 .
$$

Let $\left(\tilde{M}, \tilde{g}_{\varepsilon}\right)$ be the double cover of $\left(M, g_{\varepsilon}\right)$. Then $\lambda_{1}\left(M, g_{\varepsilon}\right)$ belongs to in $\operatorname{Spec}\left(\tilde{M}, \tilde{g}_{\varepsilon}\right)$. We denote it by $\lambda(\varepsilon)$ in brief. In Sect.1, we treat $E^{-}(\lambda)$. We denote by $v(\varepsilon)$ the non-zero first $E^{-}(\lambda)$-type eigenvalue on $\left(\tilde{M}, \tilde{g}_{\varepsilon}\right)$. We only have to compare $\lambda(\varepsilon)$ with $v(\varepsilon)$.

Proposition (C. Anné and B. Colbois [2]). Let $\left(M_{1}, g_{1}\right)$ and $\left(M_{2}, g_{2}\right)$ be two connected orientable Riemannian manifolds of the same dimension. We connected them by two tubes whose radii are both $\varepsilon$ and lengths $l_{1}$ and $l_{2}$, respectively. We denote it by $\left(M, g_{\varepsilon}\right)$ with a little smoothing at the connected parts. We express $\operatorname{Spec}\left(M, g_{\varepsilon}\right)$ as

$$
\operatorname{Spec}\left(M, g_{\varepsilon}\right)=\left\{\lambda_{0}(\varepsilon)<\lambda_{1}(\varepsilon) \leq \lambda_{2}(\varepsilon) \leq \cdots\right\}
$$

Let

$$
\left\{\mu_{0} \leq \mu_{1} \leq \mu_{2} \leq \mu_{3} \leq \cdots\right\}
$$

be the union of $\operatorname{Spec}\left(M_{1}, g_{1}\right), \operatorname{Spec}\left(M_{2}, g_{2}\right), \operatorname{Spec}_{D}\left(\left[0, l_{1}\right], \operatorname{can}\right)$ and $\operatorname{Spec}_{D}\left(\left[0, l_{2}\right]\right.$, can) (the $D$ of the $\operatorname{Spec}_{D}$ means the Dirichlet condition) counting with multiplicity.

Then for any $n$ we have

$$
\lim _{\varepsilon \rightarrow 0} \lambda_{n}(\varepsilon)=\mu_{n}
$$

Especially $\lambda_{0}(\varepsilon)=0, \mu_{0}=\mu_{1}=0$ and $\mu_{2}>0$. 
REMARK. In [2] they treat $p$-forms on $(M, g)$. It is necessary for them to assume that $\operatorname{dim} M \geq 3$. But for functions the arguments there hold good in $\operatorname{dim} M=2$. As for it see also [1].

We apply the above proposition to $\left(\tilde{M}, \tilde{g}_{\varepsilon}\right)$. Since $\lim _{\varepsilon \rightarrow 0} \lambda(\varepsilon)=0, v(\varepsilon)$ converges to some positive value which is bigger than or equal to $\mu_{2}$. The positive value depends on the equipped metrics on $\#^{n-1} \boldsymbol{R} \boldsymbol{P}^{2}, \boldsymbol{R} \boldsymbol{P}^{2}$ and the lengths of the tubes $l_{1}, l_{2}$. By the continuity of the eigenvalue in the parameter $\varepsilon$, there exists $\varepsilon_{0}>0$ such that

$$
\lambda(\varepsilon)<v(\varepsilon)
$$

for $0<\varepsilon<\varepsilon_{0}$.

Hence for all the metrics $g_{\varepsilon}$ on $M$ such that $0<\varepsilon<\varepsilon_{0}$, we have

$$
\lambda_{1}\left(M, g_{\varepsilon}\right)=\lambda(\varepsilon)=\lambda_{1}\left(\tilde{M}, \tilde{g}_{\varepsilon}\right) .
$$

\section{Example}

Here we give an example which clarifies Theorem B. Let $K(a, b)$ be the flat Klein bottle as the quotient space of $\left(\boldsymbol{R}^{2}\right.$, can) identifying by $(x, y) \mapsto(x, y+b)$ and $(x, y) \mapsto(x+a / 2,-y)$. The double cover of $K(a, b)$ is the flat torus $T(a, b)$ as the quotient space of $\left(\boldsymbol{R}^{2}\right.$, can) identifying by $(x, y) \mapsto(x+a, y)$ and $(x, y) \mapsto$ $(x, y+b)$.

The spectra of $K(a, b)$ and $T(a, b)$ are given in [4] as follows:

$$
\begin{aligned}
& \operatorname{Spec}(K(a, b))=\left\{4 \pi^{2}\left(\frac{m^{2}}{a^{2}}+\frac{n^{2}}{b^{2}}\right): m, n \in Z \text { and } n \neq 0 \text { for } m: \text { odd }\right\}, \\
& \operatorname{Spec}(T(a, b))=\left\{4 \pi^{2}\left(\frac{m^{2}}{a^{2}}+\frac{n^{2}}{b^{2}}\right): m, n \in Z\right\} .
\end{aligned}
$$

Then we have

$$
\begin{aligned}
& \lambda_{1}(K(a, b))=\left\{\begin{array}{cc}
4 \frac{\pi^{2}}{b^{2}} & \text { for } \frac{a}{2} \leq b, \\
16 \frac{\pi^{2}}{a^{2}} & \text { for } b \leq \frac{a}{2}
\end{array}\right. \\
& \lambda_{1}(T(a, b))= \begin{cases}4 \frac{\pi^{2}}{b^{2}} & \text { for } a \leq b, \\
4 \frac{\pi^{2}}{a^{2}} & \text { for } b \leq a .\end{cases}
\end{aligned}
$$


By comparing $\lambda_{1}(K(a, b))$ with $\lambda_{1}(T(a, b))$, we have

$$
\lambda_{1}(K(a, b))=\lambda_{1}(T(a, b))
$$

for $a \leq b$.

\section{References}

[1] C. Anné, Spectre du Laplacien et écrasement d'anses, Ann. Sci. Éc. Norm. Sup. 20 (1987), 271280.

[2] C. Anné et B. Colbois, Spectre du Laplacien agissant sur les $p$-formes différentielle et écrasement d'anses, Math. Ann. 303 (1995), 545-573.

[3] P. Buser, Geometry and Spectra of Compact Riemann Surfaces, Birkhauser, 1992.

[4] M. Berger P. Gauduchon et E. Mazet, Le Spectre d'une Variété Riemannienne, Lec. Notes in Math. 194 (1974), Springer.

[5] J. Cheeger, A lower bound for the smallest eigenvalue of the Laplacian, Probrems in Analysis: A Symposium in Honor of Salomon Bochoner, Princeton, 1970, pp. 195-199.

[6] S. Y. Cheng, Eigenfunctions and eigenvalues of Laplacian, Proc. Symp. in Pure Math. 27 (1975), 185-193.

[7] - Eigenfunctions and nodal sets, Comm. Math. Helv. 51 (1976), 43-55.

[ 8 ] M. J. Greenberg and J. P. Harper, Algebraic Topology, A First Course, Benjamin, 1981.

[9] H. Seifert and W. Threlfall, Lehrbuch der Topologie, Teubner, 1934.

Department of Mathematics, Tokyo Institute of Technology 2-12-1 Oh-okayama, Meguro-ku, Tokyo 152-8551, Japan

e-mail address: yoshiji@math.titech.ac.jp 